\title{
Are Extracellular Vesicles Reliable Biomarkers in Multiple
}

\section{Sclerosis?}

\section{Amoruso A, Allegretta C and Avolio C* \\ Department of Medical and Surgical Sciences, University of Foggia, Italy}

*Corresponding author: Carlo Avolio, Department of Medical and Surgical Sciences, University Of Foggia, Viale Luigi Pinto1, Foggia, Italy, Tel: +390881736080; Email: carlo.avolio@unifg.it

Abbreviations: EVs: Extracellular Vesicles; MVs: Microvesicles; EXOs: Exosomes; MS: Multiple Sclerosis; CNS: Central Nervous System; BBB: Brain Blood Barrier.

\section{Opinion}

During the last decades growing attention has been given to extracellular vesicles (EVs) released by the cells as new specialized structures for intercellular communication without direct cell contact. EVs are small membrane vesicles, commonly distinguished in microvesicles (MVs) $(100-1000 \mathrm{~nm}$ in diameter) and exosomes (EXOs) (40-100nm in diameter), which differ in size, content and mechanism of formation [1]. They are involved in different biological processes, including neuroinflammation.

The implication of EVs in the pathogenesis of neurological disorders, such as Alzheimer's, Parkinson's diseases, and Multiple Sclerosis (MS), has been already reported [2].

MS is a chronic autoimmune inflammatory demyelinating disease of the central nervous system (CNS), characterized by autoreactive lymphocyte, microglial activation and chronic neurodegeneration $[3,4]$.

The last revision of the 2010 McDonald criteria has given back absolute diagnostic relevance to the oligoclonal bands [5], certainly the most validated disease biomarker in MS, therefore to possibly find and define others represents an interesting and very attractive future goal.

In this scenario, one of the most emerging biomarkers includes EVs, immediately investigated as a potential source of information on cells involved in the pathogenesis of MS.

Although there is growing interest to study EVs, on the other hand, there are some issues that make difficult clinical application of EVs: clear and standardized characterization of the EVs, nomenclature, their biological significance and, specially, their content.

Despite these limitations, current knowledge on EVs is promising for research developments in the near future and continues to attract researcher's attention.

During last years, several groups have focused on the study of EVs in CSF and blood of patients suffering from MS.

One of the most important findings concerns the study that described increased numbers of the myeloid-derived EVs in the CSF of MS patients correlated linearly with gadolinium-enhancing MRI lesions, an index of active phase of disease [6]. Although the analysis of EVs in CSF represents an effective tool for the study of neurodegenerative diseases, analysis of this type are difficult to perform due to the limited size of the available sample, and because it is impossible to assess EVs' trend over time for ethical reasons. For this purpose, many 


\section{Annals of Immunology \& Immunotherapy}

researchers gave attention to the evaluation of EVs in more accessible biological fluids, such as peripheral blood, urine and saliva.

Increased levels of EVs released by other cell types, such as monocytes, leukocytes, platelets and endothelial cells, were observed in MS patients compared to healthy donors (HD) [7-9]. It has also been described that EVs increased the permeability of the brain blood barrier (BBB) in vitro, disrupting the endothelial barrier function [10].

Considering EVs as biomarkers of therapeutic efficacy in MS, the reduction of endothelial EVs has been observed after IFN- $\beta 1 \mathrm{~b}$ administration by Jimenez, et al. [11] as well as impairment of monocyte-EVs complex formation and transendothelial migration.

On the contrary, Saenz Cuesta, et al. [8] reported higher counts of plasma platelet MVs, lymphocyte MVs, and monocyte MVs in IFN- $\beta$ and natalizumab-treated patients.

Very recently, we have reported IFN- $\beta$ 1a, Teriflunomide and Fingolimod effects observing the reduction of monocyte-derived MVs shedding in MS patients compared to HDs [7,12].

Despite the growing interest in biological relevance of the EVs in MS pathogenesis, an open question remains about EVs content. Many reports have focused on the miRNA content, trying to define a molecular signature of disease status and progression. In particular, four exosomal miRNA were found differentially expressed in relapsing-remitting MS patients compared to HD: hsamiR-122-5p, hsa-miR-196b-5p, hsa-miR-301a-3p, and hsa-miR-532-5p [13].

In the lights of these important findings, it is clear how circulating EVs could be proposed as reliable biomarkers, providing information about clinical disease status and progression, but also therapeutic efficacy in MS. However, in this scenario, there are some technical issues, yet not solved, that make it difficult the translation of the EV analysis into the clinical practice. First of all, approaches to measure and analyse EVs in biological fluids represents the crucial point to be addressed in the future, in order to obtain a standardized procedure to separate the different types of EVs and thus to optimize also cargo analysis.

Secondly, it must be taken into account that the EV biological roles are nowadays not fully elucidated.
Certainly, EVs may represent a reliable and accessible biomarker of neuroinflammation or neurodegeneration, with a potential role in clinical practice if research will reveal their content and procedures will be developed to allow the routine detection of this content in individual samples.

Nevertheless, a lot of effort has been made and further efforts need to be planned to improve those lacking points, in order to evaluate the real power of the EVs as a novel tool in neurological diseases.

\section{References}

1. Raposo G, Stoorvogel W (2013) Extracellular vesicles: exosomes, microvesicles, and friends. J Cell Biol 200(4): 373-383.

2. Ciregia F, Urbani A, Palmisano G (2017) Extracellular Vesicles in Brain Tumors and Neurodegenerative Diseases. Front Mol Neurosci 10: 276.

3. Compston A, Coles A (2008) Multiple sclerosis. Lancet 372(9648): 1502-1517.

4. Kawachi I, Lassmann H (2017) Neurodegeneration in multiple sclerosis and neuromyelitis optica. J Neurol Neurosurg Psychiatry 88(2): 137-145.

5. Thompson AJ, Banwell BL, Barkhof F, Carroll WM, Coetzee T, et al. (2018) Diagnosis of multiple sclerosis: 2017 revisions of the McDonald criteria. Lancet Neurol 17(2): 162-173.

6. Verderio C, Muzio L, Turola E, Bergami A, Novellino L, et al. (2012) Myeloid microvesicles are a marker and therapeutic target for neuroinflammation. Ann Neurol 72(4): 610-624.

7. Blonda M, Amoruso A, Grasso R, Di Francescantonio V, Avolio C (2017) Multiple Sclerosis Treatments Affect Monocyte-Derived Microvesicle Production. Front Neurol 8: 422.

8. Saenz Cuesta M, Irizar H, Castillo Trivino T, Munoz Culla M, Osorio Querejeta I, et al. (2014) Circulating microparticles reflect treatment effects and clinical status in multiple sclerosis. Biomark Med 8(5): 653661.

9. Minagar A, Jy W, Jimenez JJ, Sheremata WA, Mauro LM, et al. (2001) Elevated plasma endothelial microparticles in multiple sclerosis. Neurology 56(10): 1319-1324. 


\section{Annals of Immunology \& Immunotherapy}

10. Marcos Ramiro B, Oliva Nacarino P, Serrano Pertierra E, Blanco Gelaz MA, Weksler BB, et al. (2014) Microparticles in multiple sclerosis and clinically isolated syndrome: effect on endothelial barrier function. BMC Neurosci 15: 110.

11. Jimenez J, Jy W, Mauro LM, Horstman LL, Ahn ER, et al. (2005) Elevated endothelial microparticlemonocyte complexes induced by multiple sclerosis plasma and the inhibitory effects of interferon-beta $1 \mathrm{~b}$ on release of endothelial microparticles, formation and transendothelial migration of monocyte- endothelial microparticle complexes. Mult Scler 11(3): 310-315.

12. Amoruso A, Blonda M, Arrigo GD, Grasso R, Di Francescantonio V, et al. (2018) Effect of fingolimod action on the release of monocyte-derived microvesicles in multiple sclerosis patients. J Neuroimmunol 15(323): 43-48.

13. Selmaj I, Cichalewska M, Namiecinska M, Galazka G, Horzelski W, et al. (2017) Global exosome transcriptome profiling reveals biomarkers for multiple sclerosis. Ann Neurol 81(5): 703-717. 